\title{
A Possible Role for Midkine in the Pathogenesis of Behçet's Syndrome
}

\author{
A. Şükrü Aynacıoğlu' ${ }^{1 *}$, Ayhan Bilir ${ }^{2}$, and M. Yakup Tuna ${ }^{3}$ \\ ${ }^{1}$ Department of Medical Pharmacology, İstanbul Aydın University, Medical Faculty, Florya Main Campus, Küçükçekmece, İstanbul, Turkey \\ 2Department of Histology and Embryology, İstanbul Aydın University, Medical Faculty, Florya Main Campus, Küçükçekmece, İstanbul, Turkey \\ ${ }^{3}$ Department of Anatomy, İstanbul Aydın University, Medical Faculty, Florya Main Campus, Küçükçekmece, İstanbul, Turkey
}

${ }^{*}$ Correspondence to: A. Şükrü Aynacıoğlu, Department of Medical Pharmacology, İstanbul Aydın University, Medical Faculty, Florya Main Campus, Küçükçekmece, 34295 İstanbul, Turkey; E-mail: as.aynacioglu@yandex.com

Received: March 20, 2018; Accepted: March 28, 2018; Published: April 1, 2018;

\begin{abstract}
Midkine (MK), a heparin-binding cytokine, is considered to be involved in disease mechanisms of several autoimmune (e.g rheumatoid arthritis, systemic lupus erythematosus and multiple sclerosis) and autoinflammatory (e.g Crohn's disease and ulcerative colitis) diseases. Behçet's syndrome (BS) is accepted as a mixed pattern disease with evidence of both acquired autoimmune component and autoinflammatory components. Therefore, our hypothesis is that MK might be overexpressed in BS patients and if so, it might serve as a disease marker in BS. Furthermore, inhibition of the hypothesized MK overexpression using MK inhibitors such as MK-aptamer might contribute to the management of BS.
\end{abstract}

\section{Introduction}

Behçet's syndrome (BS) known also as Behçet's disease (BD), was first described by Prof. Dr. Hulusi Behçet, a Turkish dermatologist in 1937 [1]. The classic trisymptom complex of this syndrome is recurrent aphthous stomatitis, genital aphthous ulcers and hypopyon-uveitis [1]. It is a chronic, relapsing-remitting inflammatory vascular disease with no pathognomonic tests. In addition to oral, genital and eye involvement multiple organ systems, including skin, gastrointestinal, vascular and neurological systems are affected [2]. The prevalence of BS is significantly higher in the Mediterranean, the Far East and Central Asia (therefore called "Silk Road Disease") compared to Europe and the United States [3-6]. Although nearly 80 years have passed since the first description of BS, the etiology and pathogenesis has not yet fully clarified. Several mechanisms, including neutrophil hyperfunction [7] and $\mathrm{T}$ cell hypersensitivity to several bacterial antigens may play a central role in the pathogenesis of BS [8].

Midkine (MK) is a growth factor (heparin-binding cytokine) that promotes a number of functions in target cells such as migration, proliferation, survival, growth, reproduction and repair, angiogenesis and gene expression [9]. $\mathrm{MK}$ is involved in the onset and/or progression of many cancers and inflammatory diseases. Therefore, it has been suggested that both MK and MK inhibitors are expected to contribute in the treatment of various diseases [10]. In addition, MK may serve as an indicator and marker in certain disorders such as rheumatoid arthritis [11].

\section{Our proposal}

Our proposal is that MK may play a role in the pathogenesis of BS. Furthermore, if MK is acting as a proinflammatory cytokine, it could be assumed that MK inhibitors may contribute to the treatment of BS.

\section{Evaluation of the proposal}

BS is a chronic inflammatory disorder caused by vasculitis that results in damage to both arteries and veins. Although the pathogenesis is not yet known, a Th1-type inflammatory reaction is seen like in some other primary vasculitides [12]. There are no biochemical tests that are specific for the diagnosis of BS, therefore the syndrome is diagnosed clinically. Some laboratory tests and imaging is done to rule out other conditions that may mimic BS. HLA B51 is used as a genetic marker for the diagnosis of BS, however it is also seen in up to $20 \%$ of the general population. The diagnosis of BS depends mostly on a good physical examination, a detailed history and presence of the typical symptoms and signs.

Recent studies showed a significant association between MK and autoimmune and autoinflammatory diseases. One of these studies showed that the plasma levels of MK and the other heparin-binding growth factor pleiotrophin were significantly higher in systemic lupus erythematosus (SLE), rheumatoid arthritis (RA) and Sjögren's syndrome (SS) patients compared with healthy controls (HCs) [13]. Furthermore, it has been demonstrated that elevated plasma midkine and pleiotrophin levels were associated with rash, anti-SSA and IL-17 in SLE patients [13].

MK participates in the migration of inflammatory leukocytes and osteoclast differentiation in RA and may be a key molecule in the pathogenesis of the disease [14]. Shindo et al. have shown that RA patients had a significantly higher serum MK level than HCs. In addition, the serum levels of MK tended to be decreased by anti-TNF therapy. They suggested that the serum MK level could be a marker of disease activity in RA and an indicator of a poor prognosis and that MK may have a role in the pathogenesis of RA via induction of inflammatory mediators [11]. 
It has been shown that normal synovial fluid and noninflammatory synovial tissue did not contain detectable MK, whereas in the inflammatory synovitis of RA and osteoarthritis (OA), MK was detected in synovial fluid, synoviocytes, and endothelial cells of new blood vessels [15]. Therefore, MK showed inflammation-associated expression in patients with RA and OA. Furthermore, MK has been demonstrated to promote chemotaxis of neutrophils and promote fibrinolysis in these cases [15].

Another study on RA showed that a chimeric-type siRNA for MK strongly inhibited postsurgical adhesion and moderately attenuated the antibody- induced arthritis in mice. Therefore, the authors suggested that MK may be an important molecular target in the treatment or prophylaxis of RA [16].

MK levels were found to be high in multiple sclerosis (MS), which is also an autoimmune disease characterized by inflammatory demyelination and neuronal damage in the central nervous system (CNS) [17]. In the review of "midkine and multiple sclerosis", Takeuchi $\mathrm{H}$. suggests that $\mathrm{MK}$ negatively regulates autoimmune tolerance by suppressing the development of DCreg and the expansion of Treg cells. Pharmacological inhibition of MK by an RNA aptamer significantly increases DCreg and Treg and ameliorates experimental autoimmune encephalomyelitis (EAE) without any detectable adverse effects. Thus, blockade of MK signaling may provide an effective therapeutic strategy against autoimmune diseases including MS [18].

Besides autoimmune diseases, $\mathrm{MK}$ is implicated also in inflammatory diseases. It has been reported that circulating MK was elevated both in quiescent and active Crohn's disease (CD) and that this elevation of MK corresponds well with disease activity and reflects the severity of inflammatory response and exacerbation of pathological angiogenesis. Furthermore, MK as a biomarker was found slightly better than that of CRP in CD [19]. Additionally, high expression of $\mathrm{MK}$ has been found in bowel inflammation in ulcerative colitis (UC) [20]. Interestingly inflammatory bowel disease is a major manifestation of BS. In fact, it can be very difficult to tell a BS patient from a patient with Crohn's disease unless extraintestinal lesions are also present in that patient [21].

In conclusion, MK plays important roles as a disease marker and as an indicator of prognosis in certain autoimmune and autoinflammatory diseases (Table 1). In addition, blockade of MK signaling may provide an effective therapeutic strategy against such disorders. We suggest that knowing the role of MK in the pathogenesis and treatment of BS, may offer new insides to the difficult management of this syndrome.

Table 1. High MK serum levels in autoinflammatory and autoimmune diseases.

\begin{tabular}{|l|l|}
\hline \multicolumn{1}{|c|}{ Autoimmune diseases } & \multicolumn{1}{c|}{ Autoinflammatory diseases } \\
\hline Rheumatoid arthritis & Chron's disease \\
\hline Systemic lupus erythematosus & Ulcerative colitis \\
\hline Sjögren's syndrome & \\
\hline Multiple sclerosis & \\
\hline \multicolumn{2}{|c|}{ Behçet's syndrome? } \\
\hline
\end{tabular}

\section{Funding: None.}

\section{Conflict of interest statement}

We declare that there are no conflicts of interest.

\section{Acknowledgement}

We would like to thank to Professor Hasan Yazici from the Department of Rheumatology, Academic Hospital, Istanbul, Turkey for valuable guidance, suggestions, and comments.

\section{References}

1. Behçet H (1937) Über rezideiverende, aphthöse, durch ein Virus verursachte Geschwüre am Mund, am Auge und an den Genitalien. Dermatol Wochenschr 46: 414-9.

2. Yurdakul S, Yazici H (2008) Behçet's syndrome. Best Pract Res Clin Rheumatol 22: 793-809. [Crossref]

3. Azizlerli G, Kose AA, Sarica R (2003) Prevalence of Behçet's disease in Istanbul, Turkey. Int J Dermatol 42: 803-806.

4. Cakir N, Dervis E, Benian O, Pamuk ON, Sonmezates N, et al. (2004) Prevalence of Behçet's disease in rural western Turkey: a preliminary report. Clin Exp Rheumatol 22: S53-55. [Crossref]

5. Zouboulis CC, Kotter I, Djawari D (1997) Epidemiological features of Adamantiades-Behçet's disease in Germany and in Europe. Yonsei Med $J$ 38: 411-422.

6. Calamia KT, Wilson FC, Icen M, Crowson CS, Gabriel SE, et al. (2009) Epidemiology and Clinical Characteristics of Behçet's Disease in the US: A Population-Based Study. Arthritis Rheum 61(5): 600-604

7. Matsumura N, Mizushima Y (1975) Leucocyte movement and colchicine treatment in Behcet's disease. Lancet 2: 813. [Crossref]

8. Hirohata S, Oka H, Mizushima Y (1992) Streptococcal-related antigens stimulate production of IL6 and interferon-gamma by T cells from patients with Behcet's disease. Cell Immunol 140(2): 410-419.

9. Muramatsu T (2014) Structure and function of midkine as the basis of its pharmacological effects. Br J Pharmacol 171(4): 814-826.

10. Muramatsu T (2011) Midkine: A Promising Molecule for Drug Development to Treat Diseases of the Central Nervous System. Current Pharmaceutical Design 17: 410-423.

11. Shindo E, Nanki T, Kusunoki N, Shikano K, Kawazoe M, et al. (2017) The growth factor midkine may play a pathophysiological role in rheumatoid arthritis. Mod Rheumatol 27: 54-59. [Crossref]

12. Melikoglu M, Kural-Seyahi E, Tascilar K, Yazici H (2008) The unique features of vasculitis in Behçet's syndrome. Clin Rev Allergy Immunol 35: 40-46. [Crossref]

13. $\mathrm{Wu}$ (2017) Elevated plasma midkine and pleiotrophin levels in patients with systemic lupus erythematosus.; 8(25): 40181-40189.

14. Maruyama (2004) Midkine, a heparin-binding growth factor, is fundamentally involved in the pathogenesis of rheumatoid arthritis. Arthritis and Rheumatism 50(5): 1420-1429.

15. Takada T, Toriyama K, Muramatsu H, Song XJ, Torii S, et al. (1997) Midkine, a retinoic acid-inducible heparin-binding cytokine in inflammatory responses: chemotactic activity to neutrophils and association with inflammatory synovitis. $J$ Biochem 122(2): 453-8.

16. Yamamoto (2006) Midkine as a molecular target: Comparison of effects of chondroitin sulfate E and siRNA. Biochemical and Biophysical Research Communications 351: 915-919.

17. Hemmer B, Archelos JJ, Hartung HP (2002) New concepts in the immunopathogenesis of multiple sclerosis. Nat Rev Neurosci 3: 291-301. [Crossref]

18. Takeuchi H (2014) Midkine and multiple sclerosis. Br J Pharmacol 171: 931-935. [Crossref]

19. Krzystek-Korpacka (2010) Circulating Midkine in Crohn's Disease: Clinical Implications. Inflamm Bowel Dis 2010; 16: 208-215.

20. Krzystek-Korpacka M, Gorska S, Diakowska D, Kapturkiewicz B, Podkowik M, et al. (2017) Midkine is up-regulated in both cancerous and inflamed bowel, reflecting lymph node metastasis in colorectal cancer and clinical activity of ulcerative colitis. Cytokine 89: 68-75. [Crossref]

21. Yazici H, Seyahi E, Hatemi G, Yazici Y (2017) Behçet syndrome: a contemporary view. Nat Rev Rheumatol. 208.

\section{Citation:}

A. Şükrü Aynacıoğlu, Ayhan Bilir, and M. Yakup Tuna (2018) A Possible Role for Midkine in the Pathogenesis of Behçet's Syndrome. Internal Med Res Open $J$, Volume 3(2): 1-2 